\title{
Estenosis isquémica tardía tras resección anterior de recto
}

\author{
J. Zuloaga, J. Arias-Díaz, S. Fernández-Díez, J. M. Balibrea y J. L. Balibrea \\ Servicio de Cirugía 2. Hospital Clínico San Carlos. Universidad Complutense de Madrid
}

\section{RESUMEN}

Existe una incidencia no despreciable de lesiones de colon de origen isquémico tras resecciones anteriores bajas por cáncer. Presentamos un caso de estenosis isquémica de colon, no relacionada con radioterapia, a los 5 años de la cirugía. Se discute la dificultad del diagnóstico diferencial con recidiva tumoral, así como el importante papel que desempeña la endoscopia en el abordaje de estos pacientes.

Palabras clave: Estenosis de colon. Rectosigmoidectomía. Cáncer colorrectal. Isquemia mesentérica. Corticosteroides.

\begin{abstract}
A considerable incidence of colonic strictures after oncologic low anterior resections has been reported. The present paper describes a colonic stricture 5 years after the surgery, and not related to radiotherapy, that required a challenging differential diagnosis with local recurrence of rectal cancer. The role of endoscopy in the management of this condition is discussed.
\end{abstract}

Key words: Colonic stricture. Rectosigmoidectomy. Colorectal cancer. Mesenteric ischemia. Steroids.

Zuloaga J, Arias-Díaz J, Fernández-Díez S, Balibrea JM, Balibrea JL. Estenosis isquémica tardía tras resección anterior de recto. Rev Esp Enferm Dig 2008; 100: 49-52.

\section{INTRODUCCIÓN}

La colitis isquémica se complica con el desarrollo de estenosis en el $10-15 \%$ de los casos (1). El edema asociado con la isquemia y la fibrosis secundaria al proceso cicatricial se postulan como los mecanismos de dicha estenosis (2).

Aunque son relativamente frecuentes las estenosis isquémicas de colon tras cirugía del aneurisma aórtico, existen muy pocos casos descritos tras resección rectal (3), estando casi todos ellos relacionados con radioterapia perioperatoria.

Aquí describimos un caso de estenosis isquémica de colon, no relacionada con radioterapia, tras una resección

Recibido: 12-07-07.

Aceptado: 23-07-07.

Correspondencia: Jaime Zuloaga Bueno. Servicio de Cirugía 2. Hospital Clínico San Carlos. C/ Doctor Martín Lagos, s/n. 28040 Madrid. e-mail: zulprada@yahoo.es anterior realizada 5 años antes, planteando un difícil diagnóstico diferencial con recidiva tumoral.

\section{CASO CLÍNICO}

Paciente varón de 65 años, fumador de 20-40 cigarrillos/día y con antecedente de ulcus duodenal. Había sido intervenido 5 años previamente por neoplasia estenosante de unión recto-sigmoidea (adenocarcinoma estadio B de Dukes, T3N0M0, estadio III-A AJCC), practicándosele resección anterior, seguida de anastomosis término-terminal mediante sutura mecánica utilizando la técnica del doble grapado. El postoperatorio de dicha intervención cursó sin complicaciones y el paciente fue seguido en consulta cada 6 meses, realizándose anualmente colonoscopia y ecografía. La imagen endoscópica de la región perianastomótica resultó normal en todas las revisiones, incluyendo la última, realizada el año anterior (a los 4 años de la intervención). 
A los 5 años de la intervención, el paciente comienza con deposiciones blandas, dolorimiento abdominal intermitente en hipogastrio y posteriormente rectorragias, por lo que es ingresado en el servicio de medicina interna, realizándosele un TAC abdominal, que no reveló hallazgos patológicos, y colonoscopia, en la que se observa una estenosis a $10 \mathrm{~cm}$ del margen anal que impide el paso del endoscopio (Fig. 1). Se realiza biopsia en la que no se aprecian signos histopatológicos de enfermedad maligna y que es informada como úlcera inespecífica.

Dado que los marcadores tumorales séricos estaban en niveles normales, y ante la ausencia de otros signos de neoplasia, se decidió repetir la endoscopia y realizar un enema opaco. La nueva endoscopia y biopsia mostraron idénticos hallazgos a los previos. En el enema opaco se observó estenosis de colon de aspecto irregular y de $8 \mathrm{~cm}$ de longitud (Fig. 2), no pudiendo descartarse con dicha imagen la posibilidad de una estenosis maligna. Ante la falta de pruebas conclusivas de recidiva tumoral, se decide un periodo de tratamiento con enemas de corticoides (budesonida, $2 \mathrm{mg}$ en enema, 2 veces al día), con el fin de reducir la inflamación y poder así completar la exploración endoscópica.

Pasado un mes, con el paciente asintomático, se repite la endoscopia, encontrándose una mucosa inflamatoria y ulcerada, tapizando una estenosis de 6-8 $\mathrm{cm}$ de longitud que ya se logra atravesar con el endoscopio, sin observarse signos de neoplasia y siendo el resto del colon de aspecto normal (Fig. 3). Las biopsias que se tomaron mostraron signos inflamatorios inespecíficos.

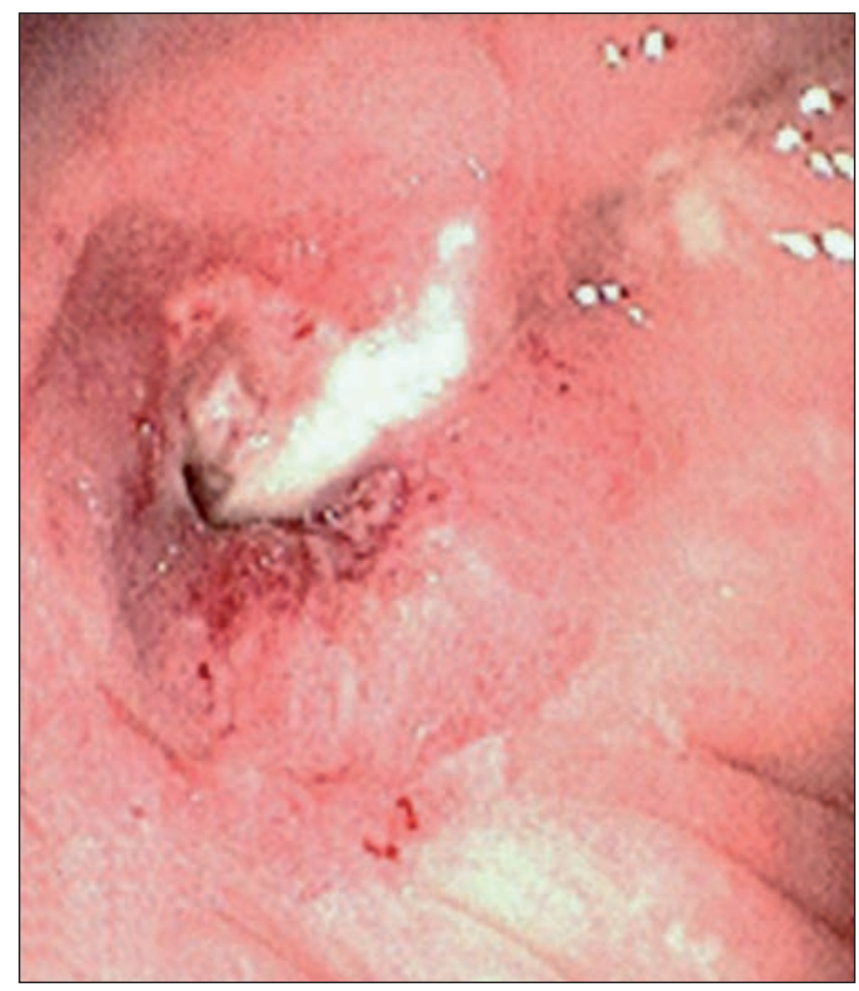

Fig. 1. Estenosis rectal que impide el paso del endoscopio.

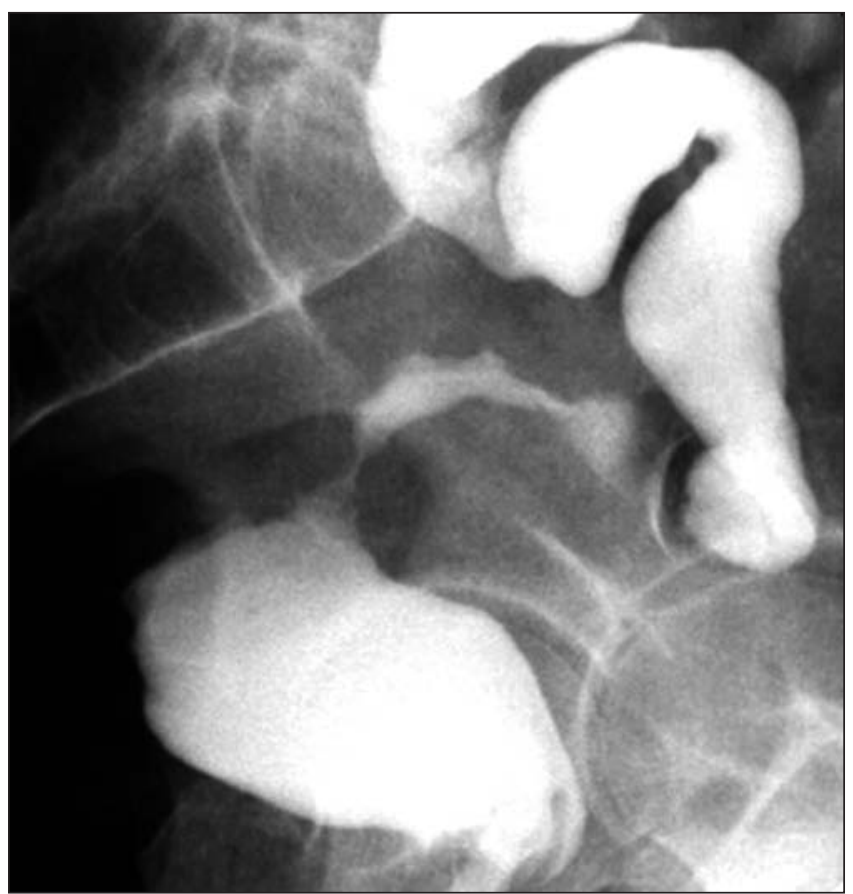

Fig. 2. Estenosis rectal (izquierda) que permite el paso del endoscopio, mostrando en su interior una mucosa de aspecto inflamatorio y ulcerado (derecha).

La estenosis es juzgada como de origen isquémico y, dado que el paciente está totalmente asintomático, se decide seguimiento periódico.

A los 6 meses de seguimiento, se detectan alteraciones electrocardiográficas, por las cuales se solicita consulta al cardiólogo. Por ergometría y coronariografía se encuentran irregularidades en descendente anterior proximal media, grave afectación del ostium del ramo diagonal, estenosis del $80 \%$ del ramo obtuso marginal y estenosis del $80 \%$ en coronaria derecha media. Se decide dilatación e implantación de un doble stent coronario, tras lo cual desaparecieron las alteraciones electrocardiográficas.

Tras 16 meses de seguimiento, el paciente presenta tránsito normal y se encuentra asintomático.

\section{DISCUSIÓN}

Las estenosis colorrectales son cuadros que a menudo se acompañan de una sintomatología no específica. Pueden reconocer múltiples causas, que podríamos dividir genéricamente en traumáticas, inflamatorias, isquémicas, tumorales o compresión extrínseca. Las causas inflamatorias podrían obedecer a su vez a cuadros criptogenéticos o autoinmunes (colitis ulcerosa, Crohn, amiloidosis, esclerodermia, etc.), infecciones, o bien fármacos, tales como antiinflamatorios no esteroideos o triptanes, entre otros.

En aquellos casos en que la estenosis aparece tras cirugía colorrectal por cáncer, la etiología que cobra mayor 

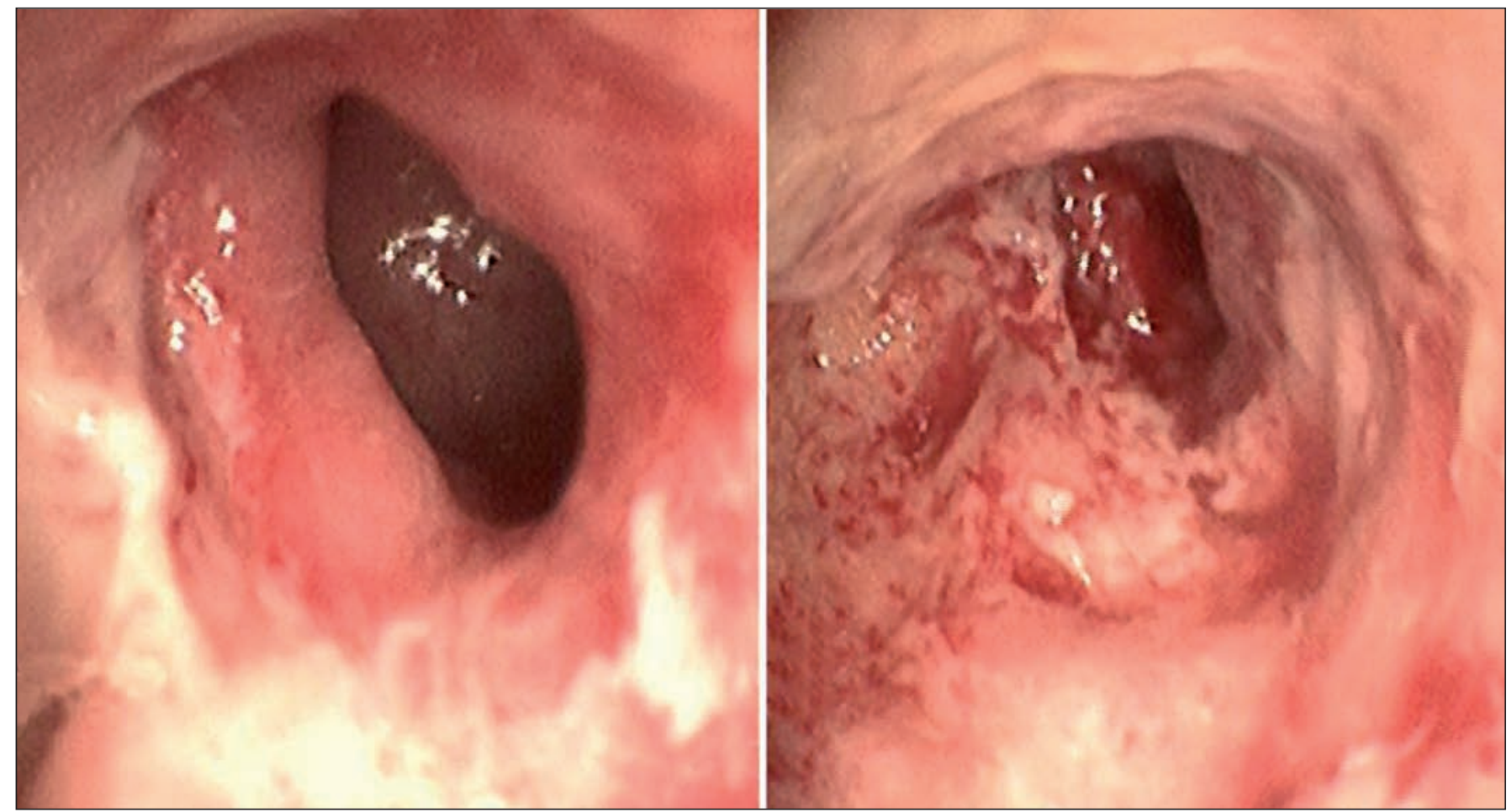

Fig. 3. Enema opaco, mostrando estenosis irregular de $8 \mathrm{~cm}$ de longitud.

importancia pronóstico-terapéutica sería la recidiva tumoral, aunque también aparecerían con mayor frecuencia causas del tipo de estenosis cicatricial (4), fuga anastomótica, compresión extrínseca y también isquemia de los segmentos cólicos adyacentes a la anastomosis.

La presentación clínica de la isquemia de colon es muy variable dependiendo de la causa subyacente, del grado de obstrucción vascular, de la rapidez de instauración de la misma, del grado de circulación colateral y de la patología asociada. Aproximadamente el $20 \%$ de los pacientes desarrollan colitis crónica por lesión isquémica irreversible, que se manifiesta por diarrea persistente, rectorragia o pérdida de peso y, endoscópicamente, como una estenosis o masa en el colon (5).

Se han descrito lesiones de colon de origen isquémico hasta en el 6\% de las resecciones anteriores bajas por cáncer (6). Un problema particularmente importante en estos pacientes sería el diagnóstico diferencial entre recidiva tumoral e isquemia ante una estenosis colorrectal de nueva aparición (7). En el caso que presentamos no fue fácil dicho diagnóstico diferencial, lográndose únicamente tras un periodo de tratamiento local con corticosteroides que facilitó el que se pudiese completar una segunda endoscopia.

Existen varias posibles causas de isquemia tras la cirugía del cáncer colorrectal. La frecuencia de colitis isquémica proximal a un tumor colorrectal parcialmente obstructivo se ha descrito entre el 1 y el 5,3\% (8). Parece que el principal factor patogénico es el aumento de presión intraluminal por encima de la estenosis neoplásica (8). En nuestro caso parece improbable la contribución de dicho factor debido a que no existían signos de obstrucción intestinal en el momento de la intervención y además la estenosis se desarrolló tardíamente tras la intervención.

$\mathrm{Si}$ bien diversos autores han atribuido a la radioterapia pre- o postoperatoria (9) una incidencia de hasta un $6 \%$ de desarrollo de estenosis tras cirugía por cáncer colorrectal, la propia técnica quirúrgica puede ser un factor patogénico importante (3). La ligadura de la arteria mesentérica inferior tras una sigmoidectomía deja la irrigación del cabo proximal totalmente dependiente de la arteria marginal, que puede a su vez estar afectada por enfermedad vascular sistémica pudiendo desembocar en isquemia. Más aún, el cabo distal pierde su irrigación procedente de la arteria rectal superior y queda dependiente por completo de las arterias hemorroidales medias. Si la sección del recto se lleva a cabo muy por encima de la reflexión peritoneal queda una zona con peligro de isquemia, el clásicamente llamado "punto crítico de Sudeck" (10). Aunque el carácter "crítico" de dicho punto haya sido discutido por diversos autores (11), alegando la existencia de una rica red anastomótica entre las arterias rectales superior, media e inferior, otros autores han confirmado que se trata de un área con escasa vascularización (12), y se han descrito casos de estenosis tras sigmoidectomía supuestamente por esta causa (3). En nuestro caso la estenosis estaba situada por encima de la anastomosis, encontrándose el cabo distal bien irrigado, por lo que parece más probable la influencia de una escasa irrigación del cabo proximal a través de la arteria marginal. Al margen de variaciones anatómicas, la causa más probable de insuficiencia de irrigación a través de dicha arcada sería la arteriosclero- 
sis. Aunque se han descrito casos raros por hiperplasia de la neoíntima (13) o vasculitis (14), la arteriosclerosis (5) es la causa más frecuente de insuficiencia vascular mesentérica, y nuestro paciente sufría arteriosclerosis sistémica, como se confirmó posteriormente por coronariografía.

Aunque la imagen colonoscópica con biopsias negativas nos puede hacer sospechar la etiología isquémica, la confirmación diagnóstica es difícil. La angiografía selectiva presenta habitualmente hallazgos poco específicos (5) y además se trata de una prueba invasiva con una incidencia no despreciable, de hasta un $15 \%$, de complicaciones significativas (15). Recientemente, algunos autores están utilizando la oximetría mucosa por espectroscopia de luz visible bajo endoscopia (16), pero su valor diagnóstico está aún por confirmar.

Para las estenosis isquémicas del colon se han sugerido diversas posibilidades terapéuticas, desde tratamiento farmacológico exclusivo a resección quirúrgica. Diversos estudios han utilizado corticoterapia, en nuestro caso la usamos en forma de enema y, aunque resultó de gran eficacia para reducir el grado de estenosis y permitir completar la endoscopia, no hemos encontrado referencias de su uso por esta vía en la estenosis isquémica de colon (17). Otros fármacos que se han sugerido con este fin han sido la PEG1 (18) o la pentoxifilina (19), administradas ambas por vía endovenosa.

También se han utilizado diversos enfoques endoscópicos para esta patología. El más habitualmente utilizado ha sido la dilatación simple, con o sin colocación de tutor metálico autoexplandible (20), seguida de electroincisión radial (21). Con dilatación se han logrado alrededor de un $70 \%$ de buenos resultados a largo plazo (22). Los tutores metálicos presentan numerosas complicaciones y constituirían una medida transitoria de resolver el problema (23). Algunos autores también han publicado buenos resultados con resección endoscópica transanal (20). En casos que no han respondido a otro tipo de medidas o bien en los que había problema de diagnóstico diferencial con tumor, se ha recurrido a resección quirúrgica del segmento afecto (24).

En el seguimiento de nuestro paciente se apreciaron anomalías electrocardiográficas que resultaron debidas a enfermedad coronaria grave y requirieron la colocación de doble stent coronario. La isquemia intestinal se asocia habitualmente con factores de riesgo tales como tabaquismo, hipertensión arterial e hiperlipidemia, todos ellos comunes con la enfermedad coronaria. De hecho, se ha comunicado hasta un $14 \%$ de infarto de miocardio tras el alta con un seguimiento medio de 4,5 años (25). Por ello, en pacientes con diagnóstico de colitis isquémica quizá se debería hacer de rutina un estudio electro-cardiográfico y probablemente también una prueba de esfuerzo.

En resumen, las estenosis isquémicas de colon tras cirugía colorrectal presentan a veces un difícil diagnóstico diferencial con recidiva tumoral y su abordaje resulta dificultoso debido a la carencia de pruebas específicas, requiriéndose con frecuencia un seguimiento estrecho del paciente como método para descartar otros procesos.

\section{BIBLIOGRAFÍA}

1. Price AB. Ischaemic colitis. Curr Top Pathol 1990; 81: 229-46.

2. Brandt LJ, Boley SJ. Colonic ischemia. Surg Clin North Am 1992; 72 (1): 203-29.

3. Yamazaki T, Shirai Y, Sakai Y, Hatakeyama K. Ischemic stricture of the rectosigmoid colon caused by division of the superior rectal artery below Sudeck's point during sigmoidectomy: Report of a case. Surg Today 1997; 27 (3): 254-6.

4. Marchena Gómez J, Ruiz de la Cuesta E, Gómez Guerra G, Vallejo Gallego I, García-Anguiano F, Hernández Romero JM. Anastomotic stricture with the EEA-Stapler after colorectal anastomosis. Rev Esp Enferm Digest 1997; 89 (11): 835-42.

5. Cappell MS. Intestinal (mesenteric) vasculopathy. II. Ischemic colitis and chronic mesenteric ischemia. Gastroenterol Clin North Am 1998; 27 (4): 827-60

6. Pahlman L, Glimelius B, Frykholm G. Ischaemic strictures in patients treated with a low anterior resection and perioperative radiotherapy for rectal carcinoma. Br J Surg 1989; 76 (6): 605-6.

7. Wolf EL, Sprayregen S, Bakal CW. Radiology in intestinal ischemia. Plain film, contrast, and other imaging studies. Surg Clin North Am 1992; 72 (1): 107-24.

8. Reeders JW, Rosenbusch G, Tytgat GN. Ischaemic colitis associated with carcinoma of the colon. Eur J Radiol 1982; 2 (1): 41-7.

9. Law WL, Choi HK, Chu KW, Tung HM. Radiation stricture of rectosigmoid treated with self-expanding metallic stent. Surgical Endoscopy 2002; 16 (7): 1106-7.

10. Van Tonder JJ, Boon JM, Becker JH, van Schoor AN. Anatomical considerations on Sudeck's critical point and its relevance to colorectal surgery. Clin Anat 2006; 20 (4): 427-7.

11. Griffiths JD. Surgical anatomy of the blood supply of the distal colon. Ann R Coll Surg Engl 1956; 19: 241-56.

12. Siddharth P, Ravo B. Colorectal neurovasculature and anal sphincter. Surg Clin North Am 1988; 68 (6): 1185-200.

13. Genta RM, Haggitt RC. Idiopathic myointimal hyperplasia of mesenteric veins. Gastroenterology 1991; 101 (2): 533-9.

14. Keating JP, King BR, Kenwright DN, Brasch HD. Vasculitis-induced colonic strictures: Report of two cases. Dis Colon Rectum 1998; 41 (10): 1316-21.

15. Kaleya RN, Boley SJ. Acute mesenteric ischemia. Crit Care Clin 1995; 11 (2): 479-512.

16. Friedland S, Benaron D, Coogan S, Sze DY, Soetikno R. Diagnosis of chronic mesenteric ischemia by visible light spectroscopy during endoscopy. Gastrointest Endosc 2007; 65 (2): 294-300.

17. Lucha PA Jr, Fticsar JE, Francis MJ. The strictured anastomosis: Successful treatment by corticosteroid injections -report of three cases and review of the literature. Dis Colon Rectum 2005; 48 (4): 862-5.

18. Abe S, Takayama T, Ohta H, et al. Successful treatment with prostaglandin E1 of ischemic colitis complicated by colonic stricture. Gastrointest Endosc 2004; 60 (1): 148-51.

19. Parra-Membrives P, Ruiz-Luque V, Escudero-Severin C, Aguilar-Luque J, Méndez-García V. Effect of pentoxifylline on the healing of ischemic colorectal anastomoses. Dis Colon Rectum 2007; 50 (3): 36975.

20. Forshaw MJ, Maphosa G, Sankararajah D, Parker MC, Stewart M. Endoscopic alternatives in managing anastomotic strictures of the colon and rectum. Techniques in Coloproctology 2006; 10 (1): 21-7.

21. Brandimarte G, Tursi A, Gasbarrini G. Endoscopic treatment of benign anastomotic colorectal stenosis with electrocautery. Endoscopy $2000 ; 32$ (6): 461-3.

22. Solt J, Hertelendy A, Szilagyi K. Long-term results of balloon catheter dilation of lower gastrointestinal tract stenoses. Dis Colon Rectum 2004; 47 (9): 1499-505.

23. Paul L, Pinto I, Gómez H, Fernández-Lobato R, Moyano E. Metallic stents in the treatment of benign diseases of the colon: Preliminary experience in 10 cases. Radiology 2002; 223 (3): 715-22.

24. Simi M, Pietroletti R, Navarra L, Leardi S. Bowel stricture due to ischemic colitis: Report of three cases requiring surgery. Hepatogastroenterology 1995; 42 (3): 279-81.

25. Huguier M, Barrier A, Boelle PY, Houry S, Lacaine F. Ischemic colitis. Am J Surg 2006; 192 (5): 679-84. 